\title{
Damping-Growth Transition for Ion-Acoustic Waves in a Density Gradient
}

\author{
D'Angelo, N.; Michelsen, Poul; Pécseli, Hans
}

Published in:

Physical Review Letters

Link to article, DOI:

10.1103/PhysRevLett.34.1214

Publication date:

1975

Document Version

Publisher's PDF, also known as Version of record

Link back to DTU Orbit

Citation (APA):

D'Angelo, N., Michelsen, P., \& Pécseli, H. (1975). Damping-Growth Transition for lon-Acoustic Waves in a Density Gradient. Physical Review Letters, 34(19), 1214-1216. https://doi.org/10.1103/PhysRevLett.34.1214

\section{General rights}

Copyright and moral rights for the publications made accessible in the public portal are retained by the authors and/or other copyright owners and it is a condition of accessing publications that users recognise and abide by the legal requirements associated with these rights.

- Users may download and print one copy of any publication from the public portal for the purpose of private study or research.

- You may not further distribute the material or use it for any profit-making activity or commercial gain

- You may freely distribute the URL identifying the publication in the public portal

If you believe that this document breaches copyright please contact us providing details, and we will remove access to the work immediately and investigate your claim 


\title{
Damping-Growth Transition for Ion-Acoustic Waves in a Density Gradient
}

\author{
N. D'Angelo \\ Danish Space Research Institute, Lyngby, Denmark, and Danish Atomic Energy Commission \\ Research Establishment Ris $\phi$, Roskilde, Denmark \\ and \\ P. Michelsen and H. L. Pécseli \\ Association EURATOM-Atomenergikommissionen, Danish Atomic Energy Commission \\ Research Establishment Ris $\phi$, Roskilde, Denmark
}

(Received 18 February 1975)

\begin{abstract}
A damping-growth transition for ion-acoustic waves propagating in a nonuniform plasma ( $e$-folding length for the density $l_{n}$ ) is observed at a wavelength $\lambda \sim 2 \pi l_{n}$. This result supports calculations performed in connection with the problem of heating of the solar corona by ion-acoustic waves generated in the solar photosphere.
\end{abstract}

The behavior of ion-acoustic perturbations propagating, along a density gradient, in a highly ionized, nonuniform plasma is of considerable interest in astrophysics and in geophysics. One example of this effect is provided by the problem of coronal heating.

Heating of the solar corona through Landau damping of ion-acoustic perturbations generated in the photosphere was discussed previously. ${ }^{1,2}$ In the solar corona two competing effects have, in general, to be considered. One is the Landau damping of the ion-acoustic perturbations in a plasma of approximately equal ion and electron temperatures $\left(T_{i} \approx T_{e}\right)$, and the other is a growth in the relative wave amplitude $(\tilde{n} / n)$ when the perturbations move outward to more and more tenuous regions. A fair guess ${ }^{1,2}$ was that Landau damping should be the dominant effect for wavelengths shorter than the scale height of the corona, whereas growth should occur for longer wavelengths. Calculations $\mathrm{s}^{3,4}$ have shown that in an isothermal (and $T_{i} \approx T_{e}$ ) exponential atmosphere acted upon by the solar gravity, growth occurs for wavelengths $\lambda>2 \pi l_{n}$, where $l_{n}$ is the scale height. For $\lambda<2 \pi l_{n}$ the ion-acoustic perturbations are Landau damped in much the same way as in a plasma of uniform density.

In the laboratory, the hydrodynamic behavior of ion-acoustic perturbations in a nonuniform plasma has been observed by Doucet, Jones, and Alexeff. ${ }^{5}$ Since their plasma, however, had a $T_{e} / T_{i}$ ratio $\gg 1$, Landau damping could not be an important process.

The present, preliminary report contains the (to the best of our knowledge) first experimental demonstration of a transition from (hydrodynamic) growth to (Landau) damping of ion-acoustic perturbations, as the wavelength is varied from below to above $\sim 2 \pi l_{n}$. In a laboratory experiment the situation is, of course, different from that of the solar corona also in the sense that the zeroorder density gradient cannot be produced by gravity. As explained below, the density gradient in the experiment was produced by a combination of an axial electric field and radial plasma losses. The overall agreement between our measurements and the results of the calculations performed for the case of an isothermal atmosphere held by gravity (Refs. 3 and 4) indicates that these results apply to ion-acoustic wave behavior in density gradients no matter how the gradients are produced.

The experiment was conducted in a cesium plasma column of a conventional, single-ended $Q$ device, ${ }^{6}$ in which $T_{i} \approx T_{e}$. The plasma density was generally in the range (1 to 5$) \times 10^{9} \mathrm{~cm}^{-3}$, with the neutral gas pressure $\lesssim 10^{-5} \mathrm{~mm} \mathrm{Hg}$. A (variable) density gradient along the direction of the magnetic field lines was produced by surrounding a portion of the plasma column $(\sim 3 \mathrm{~cm}$ diameter) with a metal tube of $3.5 \mathrm{~cm}$ in diameter biased to -30 to $-100 \mathrm{~V}$ with respect to the generating plate of the $Q$ device (see, for instance, Roberson, Ratner, and Hirshfield ${ }^{7}$ ). Ion-acoustic waves were excited, in much the same manner as, e.g., by Wong, Motley, and D'Angelo, ${ }^{8}$ by a grid immersed in the plasma (normal to the axis of the column) between the generating plate and the metal tube. The steepness of the axial density profile (density decreasing from grid to tube) could be varied by varying the negative tube bias, more negative voltages on the tube giving rise to steeper density profiles. For a fixed tube voltage, smaller values of the confining magnetic field 
gave rise to steeper density profiles.

Measurements were performed by means of a Langmuir probe, movable over a distance of $\sim 25 \mathrm{~cm}$ along the axis of the plasma column. The exposed surface of the probe was a flat metal disc, $5 \mathrm{~mm}$ in diameter, oriented normal to the column axis. The zero-order axial density profile is obtained most easily through a measurement of the probe floating-potential profile and the relation $\kappa T_{e} \partial n / \partial x=e n \partial \varphi / \partial x$ [e.g., Refs. 6 and 8$]$ between density and potential gradients. The same 5-mm-diam probe was used to measure the axial plasma flux as a function of position along the plasma column. The flux was observed to decrease as the probe was moved away from the generating plate, thus showing the presence of radial plasma losses. We believe that these losses are produced in part by an ion "scrape-off" (the Larmor radius can be as large as $\sim 1 \mathrm{~cm}$ ) and in part by enhanced radial diffusion. When the zero-order plasma behavior is analyzed on the basis of continuity and momentum equations, including a radial loss term, agreement is found with the measured zero-order quantities. Clearly, an axial density gradient is associated with an axial electric field, the electrons being in Boltzmann equilibrium.

The wave measurements were performed with the probe electrically floating, since $\tilde{n} / n=e \tilde{\varphi} /$ $\kappa T_{e}$ for waves of the type we are here concerned with ( $\tilde{n}$ is the perturbed density and $\tilde{\varphi}$ the perturbed potential). The results of the wave measurements are summarized in Fig. 1. At any

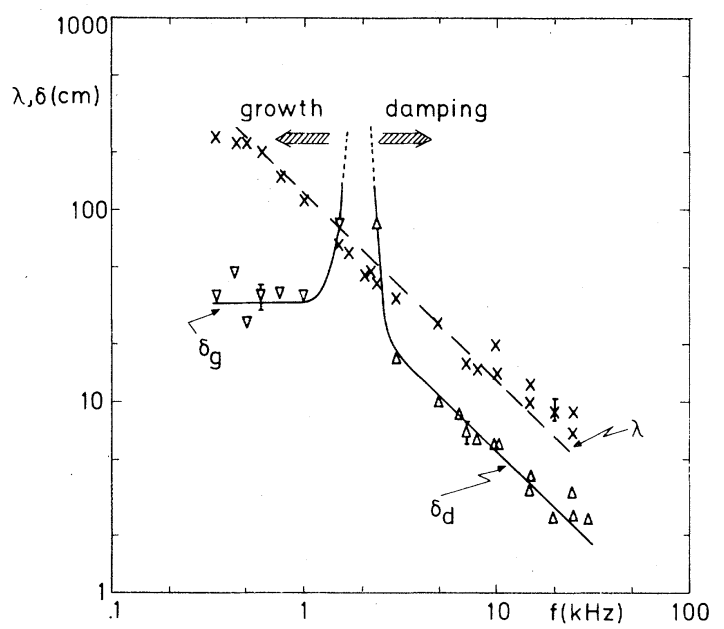

FIG. 1. The wavelength, $\lambda$, and the damping, $\delta_{d}$, or growth, $\delta_{g}$, distance versus the wave frequency, $f$. $\left[n=(1-5) \times 10^{9} \mathrm{~cm}^{-3}, B=2000 \mathrm{G}\right.$. $]$ The bias on the metal tube was $-30 \mathrm{~V}$. frequency, $f$, the corresponding wavelength is indicated by a cross. Above $f \sim 2 \mathrm{kHz}$ the waves are damped as they propagate away from the grid, and $\delta_{d}$ (with triangles pointing upward) is the damping distance. Below $f \sim 2 \mathrm{kHz}$ the waves grow; $\delta_{g}$ (with triangles pointing downward) is the growth distance. The measurements of Fig. 1 were performed with a bias on the metal tube of $-30 \mathrm{~V}$, which corresponds to an $e$-folding length for the axial density profile of $\sim 12 \mathrm{~cm}$. The confining magnetic field was $2000 \mathrm{G}$.

We note the following from Fig. 1: (a) $f \lambda=$ const $\approx 1.2 \times 10^{5} \mathrm{~cm} / \mathrm{sec}$, as expected for the phase velocity of ion-acoustic perturbations in this plasma. (b) For $f \geq 3 \mathrm{kHz}$, the ratio $\delta_{d} / \lambda \simeq 0.5$, independent of frequency (a well known feature of Landau-damped ion-acoustic waves, when $T_{i}$ $\approx T_{e}$ ). (c) For $f \leqslant 1 \mathrm{kHz}$, the growth distance $\delta_{g}$ is independent of frequency (for $350 \mathrm{~Hz} \leqslant f \leqslant 1000$ $\mathrm{Hz}$ ), as predicted by the hydrodynamic model (e.g., Refs. 1-5). Also, $\delta_{g} \approx 2 l_{n}\left(2 l_{n} \approx 24 \mathrm{~cm}\right)$. (d) At $f$ near $2 \mathrm{kHz}, \delta_{d}$ and $\delta_{g}$ become very large, as expected at the frequency of transition between growth and damping.

In order to fortify the results in Fig. 1, we show in Fig. 2 those obtained in a somewhat different experiment. The ratio, $R$, between the wave amplitude at a distance $x_{2}=19 \mathrm{~cm}$ from the exciting grid and the wave amplitude at $x_{1}=5 \mathrm{~cm}$ is plotted versus wave frequency. The numbers in brackets indicate the bias (in volts) on the metal tube. Remember that a more negative bias corresponds to a steeper axial density gradient. Clearly, as expected, the transition between growth and damping $(R=1)$ moves to lower fre-

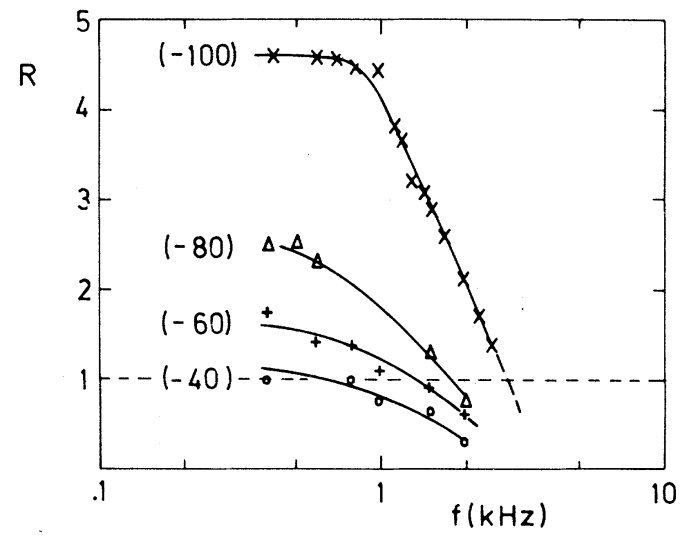

FIG. 2. The ratio, $R$, of wave amplitude at $x_{2}=19 \mathrm{~cm}$ from the grid to the wave amplitude at $x_{1}=5 \mathrm{~cm}$ versus the wave frequency. In brackets is the tube bias in volts $\left[n=(1-5) \times 10^{9} \mathrm{~cm}^{-3}, B=3000 \mathrm{G}\right]$. 
quencies as the steepness of the density profile is reduced. Note that in Fig. 2 the magnetic field strength differs from that in Fig. 1.

In addition, the relation between $l_{n}$ and the (growth-damping) transition wavelength, $\lambda_{c}$, was observed to be $\lambda_{c} \simeq 2 \pi l_{n}$, by varying $l_{n}$ by a factor $\sim 4$. For instance, in the conditions of Fig. 2, values of $\lambda_{c} \simeq 40,70,100$, and $180 \mathrm{~cm}$ corresponded to values of $l_{n} \simeq 6,11,18$, and $25 \mathrm{~cm}$, respectively.

Great care was exercised, particularly at the low-frequency end of the range of frequencies explored, to make sure that no wave reflection from the open end of the plasma column took place. This point was checked (a) by the actual wave phase measurements, which under all circumstances showed that we were indeed dealing with propagating waves, and (b) by altering the boundary conditions at the open end, without affecting the observed wave behavior. This fact is not surprising since the ions are not reflected from the end of the column.

It may be appropriate to mention here that, with the steepest density profiles $\left(V_{\text {tube }} \lesssim-100 \mathrm{~V}\right)$, we have observed the generation of harmonics when a growing wave, in propagating away from the grid, produces a density modulation comparable with the zero-order density. This is due to a rectifier (or "diode") effect which occurs at $\tilde{n}$ / $n \sim 100 \%$ (the total density cannot become negative) and which may be looked upon, since $\tilde{v} \simeq C_{s} \tilde{n} / n$ ( $\tilde{v}$ is the perturbed velocity and $C_{s}$ the ion-acoustic speed), as the ejection, at the applied frequency, of plasma "blobs" from the low-density end of the column.

Finally a comment as to our results against the background of a controversy which has arisen in recent years among $Q$-machine workers (see Motley $^{9}$ for a summary), about the importance of phase-mixing of freely streaming particles versus collective interaction. The present results seem to emphasize the importance of collective interaction in ion-acoustic waves in inhomogeneous plasmas with $T_{i} \simeq T_{e}$. The hydrodynamic growth we have measured at low frequencies is mainly due to the interaction between the zero-order electric field associated with the density gradient and the particles in the wave perturbation. A pure "free-streaming" model does not appear to provide an immediate, adequate explanation of this result, since the zero-order distribution and the perturbed distribution at the grid must be equally affected by the radial losses. Note, however, that the concept of "freestreaming" has only been applied to uniform plasmas.

We thank M. Nielsen and B. Reher for their skillful technical assistance.

${ }^{1}$ N. D'Angelo, Astrophys. J. 154, 401 (1968).

${ }^{2}$ N. D'Angelo, Solar Phys. 7,321 (1969).

${ }^{3}$ D. Parkinson and K. Schindler, J. Plasma Phys. $\underline{3}$, 13 (1969).

${ }^{4}$ C. H. Liu, J. Plasma Phys. $\underline{4}, 617$ (1970).

${ }^{5}$ H. J. Doucet, W. D. Jones, and I. Alexeff, Phys. Fluids 17, 1738 (1974).

${ }^{6}$ N. Rynn and N. D'Angelo, Rev. Sci. Instrum. $\underline{31}$, 1326 (1960).

${ }^{7}$ C. W. Roberson, A. S. Ratner, and J. L. Hirshfield, Phys. Rev. Lett. 31, 1041 (1973).

${ }^{8}$ A. Y. Wong, R. W. Motley, and N. D'Angelo, Phys. Rev. 133, A436 (1964).

${ }^{9} \mathrm{R}$. W. Motley, Q-machines (Academic, New York, 1975). 